\title{
Lung adenocarcinoma subtyping using preoperative biopsy and cytology specimens
}

\author{
Hiroyuki Miura $^{1}$, Jun Miura ${ }^{2}$ and Hiroshi Hirano ${ }^{3 *}$ \\ ${ }^{1}$ Department of Thoracic Surgery, Akiru Municipal Medical Center, Japan \\ ${ }^{2}$ Department of Surgery, Kyorin University School of Medicine, Japan \\ ${ }^{3}$ Department of Pathology, Hachioji Medical Center of Tokyo Medical University, Japan
}

\begin{abstract}
Background and objective: The $4^{\text {th }}$ edition of the WHO Classification of Tumors of the Lung, Pleura, Thymus and Heart states that adenocarcinomas should be classified according to the predominant pattern. Furthermore, the text mentions that adenocarcinoma subtyping should be performed even in biopsy specimens and cytological specimens.
\end{abstract}

Methods: Among adenocarcinoma patients who underwent surgical resection from 2015 to 2016, 53 patients who were diagnosed as having adenocarcinoma based on preoperative bronchoscopic examination were retrospectively analyzed. An attempt was made to perform lung adenocarcinoma subtyping preoperatively using transbronchial lung biopsy (TBLB) specimens, brush cytology and wash cytology specimens.

Results: The predictive value of TBLB specimens was $56.2 \%$. Those from brush cytological specimens and wash cytological specimens were $64.1 \%$ and $51.9 \%$, respectively.

Conclusion: The concordance rate between the preoperative specimens and the resected specimens should be known at each institution. This information can be used in subtype estimation for patients with tumors that cannot be resected.

\begin{abstract}
Abbreviations: TBLB: Transbronchial Lung Biopsy, H\&E: Hematoxylin and Eosin, NSCC-NOS: Non-Small Cell Carcinoma, not otherwise specified, Pap: Papillary Adenocarcinoma, Sol: Solid Adenocarcinoma, Lep: Lepidic Adenocarcinoma, Muc: Mucinous Adenocarcinoma, MIA: Minimally Invasive Adenocarcinoma, Micropap: Micropapillary Adenocarcinoma, Enteric: Enteric Adenocarcinoma, Neg: Negative
\end{abstract}

\section{Introduction}

According to the $3^{\text {th }}$ edition of the WHO Classification of Tumors of the Lung, Pleura, Thymus and Heart, most adenocarcinoma subtypes are divided into mixed subtypes [1]. This mixed subtype means less to physicians because this enormous group cannot show each prognostic factor or each of the tumor characteristics. On the other hand, the $4^{\text {th }}$ edition of the WHO Classification of Tumors of the Lung, Pleura, Thymus and Heart states that adenocarcinomas should be classified according to the predominant pattern [2]. In particular, solid subtypes and micropapillary subtypes showed worse prognoses [3-6]. Therefore, this classification is very useful for physicians in determining a therapeutic strategy. Furthermore, the text mentions that adenocarcinoma subtyping should be performed even in biopsy specimens and cytological specimens [2]. This adenocarcinoma subtyping using biopsy specimens and cytological specimens is also recommended in the $8^{\text {th }}$ edition of the Classification of Lung Cancers published by the Japan Lung Cancer Society [7]. Transbronchial examinations can only estimate the tumor surface that involves the bronchus or bronchioles. Naturally, specimens from transbronchial examinations do not represent the entire tumor. If adenocarcinoma subtypes can be presumed based on the part of the tumor, the classification may be very useful especially in inoperable cases.

\section{Patients and methods}

Among adenocarcinoma patients who underwent surgical resection from 2015 to 2016, 53 patients who were diagnosed with adenocarcinoma based on preoperative bronchoscopic examinations were retrospectively analyzed. An attempt was made to perform lung adenocarcinoma subtyping preoperatively using transbronchial lung biopsy (TBLB) specimens, brush cytology and wash cytology specimens according to the 2015 World health Organization classification system [2], and the subtyping results were compared with the final subtyping diagnosis made using resected specimens. The pathological specimens were assessed by board certified pathologists, and the cytological specimens were assessed by board certified cytopathologists without consultation between the two parties. The consent regarding this research has been obtained by all patients.

In the bronchoscopic procedure, the TBLB was performed first, followed by brush cytology and wash cytology in sequential order. TBLB specimens were fixed with formalin, embedded in paraffin, and subjected to H\&E staining. The cytological specimens were fixed with 95\% ethyl alcohol and Papanicolaou staining was performed.

${ }^{\star}$ Correspondence to: Hiroyuki Miura, Department of Thoracic Surgery, Akiru Municipal Medical Center, 78-1 Hikida, Akiruno city Tokyo, Japan, E-mail: arowana@akiru-med.jp

Key words: lung cancer, adenocarcinoma, subtyping, TBLB, cytology

Received: June 08, 2018; Accepted: July 02, 2018; Published: July 05, 2018 
Ethics approval was not received for this human study because this was a study of the results of routine examinations and informed consents of a retrospective survey of the results were obtained from all patients before the examination. There was no extra burden to the patients. All adult participants provided written informed consent to using the examination results and the informed consents were recorded in each medical record.

\section{Results}

\section{Transbronchial lung biopsy}

Among the 53 adenocarcinoma patients, TBLB was performed in 52 patients and a positive diagnosis was obtained in 48 patients. Five invasive mucinous adenocarcinomas, one micropapillary adenocarcinoma and one enteric adenocarcinoma were classified accurately. Among the 27 papillary adenocarcinomas, 15 were classified as papillary adenocarcinomas accurately. However, 12 tended to be classified as types with poor differentiation, such as acinar or solid adenocarcinomas. On the contrary, 3 out of 4 solid adenocarcinomas tended to be classified as types with better differentiation, such as papillary adenocarcinoma and acinar adenocarcinomas. Lepidic growths were not accurately detected and were classified as papillary adenocarcinomas or acinar adenocarcinomas. The predictive value was $56.2 \%$ (Table 1).

\section{Brush cytology}

Among the 53 adenocarcinoma patients, brush cytology was performed in 52 patients and a positive diagnosis was obtained in 39 patients. Three invasive mucinous adenocarcinomas were classified accurately. Nineteen out of twenty-four papillary adenocarcinomas were classified accurately. Four were classified as types with poor differentiation, lepidic growth was identified in one patient. Among the five solid adenocarcinomas, one was difficult to subtype, and one was classified as non-small cell carcinoma not otherwise specified due to poor differentiation. Only two patients were accurately classified. None of the acinar adenocarcinomas were classified accurately. The predictive value was $64.1 \%$ (Table 2).

\section{Wash cytology}

Among the 53 adenocarcinoma patients, brush cytology was performed in all patients and a positive diagnosis was obtained in 27 patients. In seven patients, a subtype could not be identified. Only a diagnosis of adenocarcinoma could be made in these patients. For one, the only diagnosis made was NSCC-NOS. Twelve out of sixteen positive papillary adenocarcinomas were classified accurately. None of the acinar adenocarcinomas were classified. Only one out of five positive solid adenocarcinomas were classified accurately. Three other solid adenocarcinomas were classified as types with better differentiation. The predictive value was $51.9 \%$ (Table 3 ).

Table 1. Adenocarcinoma subtyping difference between TBLB and resected specimen.

\begin{tabular}{|c|c|c|c|c|c|c|c|c|c|c|}
\hline TBLB & Pap. & Acinar & Sol. & G2 & Muc. & Micro p. & Enteric & Neg./ Susp. & Not $p$. & Total \\
\hline Pap. & 15 & 6 & 5 & 1 & & & & 2 & & 29 \\
\hline Acinar & 1 & 4 & & & & & & & 1 & 6 \\
\hline Solid & 2 & 1 & 1 & & & & & 2 & & 6 \\
\hline Lepidic/MIA & 4 & 1 & & & & & & & & 5 \\
\hline Mucin. & & & & & 5 & & & & & 5 \\
\hline Micro pap. & & & & & & 1 & & & & 1 \\
\hline Enteric & & & & & & & 1 & & & 1 \\
\hline Total & 22 & 12 & 6 & 1 & 5 & 1 & 1 & 4 & 1 & 53 \\
\hline
\end{tabular}

Concordance rate: $27 / 48=56.2 \%$

Table 2. Adenocarcinoma subtyping difference between brush cytology and resected specimen.

\begin{tabular}{|c|c|c|c|c|c|c|c|c|c|c|}
\hline Brush & Pap. & Acinar & Sol. & Lep. & Muc. & ad & NOS & Neg./Susp & Not p. & Total \\
\hline Pap. & 19 & 2 & 2 & 1 & & & & 4 & 1 & 29 \\
\hline Acinar & 2 & & 1 & & & & & 3 & & 6 \\
\hline Solid & 1 & & 2 & & & 1 & 1 & 1 & & 6 \\
\hline Lepidic/MIA & 2 & & & 1 & & 1 & & 1 & & 5 \\
\hline Mucin. & & & & & 3 & & & 2 & & 5 \\
\hline Micro pap. & & & & & & & & 1 & & 1 \\
\hline Enteric & & & & & & & & 1 & & 1 \\
\hline Total & 24 & 2 & 5 & 2 & 3 & 2 & 1 & 13 & 1 & 53 \\
\hline
\end{tabular}

Concordance rate: $25 / 39=64.1 \%$

Table 3. Adenocarcinoma subtyping difference between wash cytology and resected specimen.

\begin{tabular}{|c|c|c|c|c|c|c|c|c|}
\hline Path. & Pap. & Acinar & Sol. & Muc. & ad & NOS & Neg./Susp. & Total \\
\hline Pap. & 12 & & & & 4 & & 13 & 29 \\
\hline Acinar & 1 & & 1 & & 1 & & 3 & 6 \\
\hline Solid & 2 & 1 & 1 & & & 1 & 1 & 6 \\
\hline Lepidic/MIA & & & & & 2 & & 3 & 5 \\
\hline Mucin. & & & & 1 & & & 4 & 5 \\
\hline Micro pap. & & & & & & & 1 & 1 \\
\hline Enteric & & & & & & & 1 & 1 \\
\hline Total & 15 & 1 & 2 & 1 & 7 & 1 & 26 & 53 \\
\hline
\end{tabular}

Concordance rate: $14 / 27=51.9 \%$ 


\section{Discussion}

Invasive mucinous adenocarcinoma presents with distinctive features such as goblet cell-like mucous rich tumor cell clusters. Therefore, invasive mucinous adenocarcinoma was classified accurately using cytological specimens instead of pathological specimens. In only one case, enteric adenocarcinoma was also correctly diagnosed using biopsy specimens due to its characteristic morphology. If cytological specimens from the cell clusters of tall columnar cells exist, enteric adenocarcinoma could also be correctly diagnosed. However, with these cytological features, the initial diagnosis would be metastatic tumors originating from colorectal cancer without immunohistochemical staining.

Papillary adenocarcinoma tends to be classified as a subtype with poorer differentiation such as acinar or solid adenocarcinoma in biopsy specimens than cytological specimens. Papillary adenocarcinoma was classified more accurately in cytological specimens than in pathological specimens. The reason could be that the three-dimensional structure was more emphasized in the cytological specimens. On the contrary, solid adenocarcinoma tends to be judged as a better differentiated subtype. since the differentiated structures, such as those in papillary or acinar adenocarcinoma, were emphasized in the smaller specimens. Especially in cytological specimens, acinar adenocarcinoma could not be correctly identified because papillary structures were easily detected instead of an acinar structure. Adenocarcinoma subtypes could be determined in many patients using wash cytology specimens. Only a diagnosis of adenocarcinoma was made based on eccentric nuclei and a three-dimensional structure. Excluding this study, there are some reports in which wash cytology becomes a trigger for suspecting micropapillary adenocarcinoma. A determination using the total of findings from biopsies, brush cytologies and wash cytologies is preferred. None of the lepidic adenocarcinomas could be correctly diagnosed using the biopsy specimens. since the papillary structures were significantly more prominent. In cytological specimens, lepidic growth was suspected based on sheet-like cell clusters with mild atypia. Certainly, differentiation between adenocarcinoma in situ, minimally invasive adenocarcinoma and lepidic adenocarcinoma would not be feasible only using cytological or biopsy specimens. Therefore, references to diagnostic imaging should be made prior to making a diagnosis.

There are few studies in which preoperative adenocarcinoma subtyping was performed. Haung et al. tried to perform adenocarcinoma subtyping using CT-needle biopsy specimens or EVUS specimens [8]. Despite the more central specimens compared with those used in our study, the concordance rate between resected specimens and biopsy specimens was $58.6 \%$ ( 75 out of 128 specimens). The concordance rate for solid adenocarcinoma was $30 \%$ (3 out of 10 specimens). None of the cases were identified correctly as micropapillary adenocarcinoma. Whether or not solid adenocarcinoma and micropapillary adenocarcinoma exist is a poor prognostic factor, and the concordance rate was only $16.5 \%$. Bittar et al. [9] performed adenocarcinoma subtyping using intraoperative frozen sections of stage I lung adenocarcinoma. The intraoperative diagnosis and the diagnosis from permanent sections were concordant in 78 out of 112 patients $(69.7 \%)$. They assumed this discrepancy as being due to inadequate sampling and poor quality frozen sections. Rodriguez et al. [10] tried to perform cytological adenocarcinoma subtyping. Only 26 out of the 66 cases were concordant between the predominant pattern on resection and cytology specimens. They also pointed out that the concordance rate increased in specimens that had more than 200 tumor cells.

\section{Conclusions}

The concordance rate between preoperative specimens and resected specimens should be known at each institution. This information can be used in subtype estimation for patients with tumors that cannot be resected.

\section{Conflict of interest}

The authors have declared that no conflict of interest exists.

\section{References}

1. Travis WD, Brambilla E, Muller-Hermelink HK, Harris CC. Adenocarcinoma, World Health Organization Classification of Tumors. ( $3^{\text {rd }}$ Edn). Pathology and Genetics of Tumors of the lung, Pleura, Thymus and heart. WHO Lyon, France, 2003; 35-44.

2. Travis WD, Brambilla E, Burke AP, Marx A, Nicholson AG. Adenocarcinoma, World Health Organization Classification of Tumors. ( $4^{\text {th }}$ Edn). Pathology and Genetics of Tumors of the lung, Pleura, Thymus and heart. International Agency for Research on Cancer, Lyon, France, 2015; 26-46

3. Zhao Y, Wang R, Shen X, Pan Y, Cheng C, et al (2016) Minor Components of Micropapillary and Solid Subtype in Lung Adenocarcinoma are Predictors of Lymph Node Metastasis and Poor Prognosis. Ann Surg Oncol 23: 2099-2105. [Crossref]

4. Hirano H, Maeda H, Takeuchi Y, Susaki Y, Kobayashi R, et al. (2014) Lymphatic invasion of micropapillary cancer cells is associated with a poor prognosis of pathological stage IA lung adenocarcinomas. Oncol Lett 8: 1107-1111. [Crossref]

5. Ujiie H, Kadota K, Chaft JE, Uitrago D, Shima CS, et al. (2015) Solid Predominan Histologic Subtype in Resected stage I Lung Adenocarcinoma Is an Independent predictor of Early, Extrathoracic, Multisite Recurrence and Poor Postrecurence survival. J Clin Oncol 33: 2877-2884. [Crossref]

6. Tsutsumida H, Nomoto M, Goto M, Kitajima S, Kubota I, et al. (2007) A micropapillary pattern is predictive of a poor prognosis in lung adenocarcinoma, and reduced surfactant apoprotein A expression in the micropapillary pattern is an excellent indicator of a poor prognosis. Mod Pathol 20: 638-647. [Crossref]

7. The Japan Lung Cancer Society. General Rule for Clinical and Pathological Record of Lung Cancer. Kanehara, Tokyo 2017. (Text in Japanese)

8. Huang KY, Ko PZ, Yao CW, Hsu CN, Fang HY, et al. (2017) Inaccuracy of lung adenocarcinoma subtyping using preoperative biopsy specimens. J Thorac Cardiovasc Surg 154: 332-339. [Crossref]

9. Bittar HE, Incharoen P, Althouse AD, Dacic S (2015) Accuracy of the IASLC/ATS ERS histological subtyping of stage I lung adenocarcinoma on intraoperative frozen sections. Mod Pathol 28: 1058-1063.

10. Rodriguez EF, Monaco SE, Dacic S (2013) Cytological subtyping of lung adenocarcinoma by using the proposed International Association for the Study of lung Cancer/ American Thoracic Society/European Respiratory Society (IASLC/ATS/ERS) adenocarcinoma classification. Cancer Cytopathol 121: 629-637. [Crossref]

Copyright: (C2018 Miura H. This is an open-access article distributed under the terms of the Creative Commons Attribution License, which permits unrestricted use, distribution, and reproduction in any medium, provided the original author and source are credited. 